\title{
Signal Selection and Follow-Up in Pharmacovigilance
}

\author{
Ronald H.B. Meyboom,1,2,3 Marie Lindquist, ${ }^{1}$ Antoine C.G. Egberts 2,4 \\ and I. Ralph Edwards 1 \\ 1 The Uppsala Monitoring Centre, Uppsala, Sweden \\ 2 Department of Pharmacoepidemiology and Pharmacotherapy, Utrecht Institute for \\ Pharmaceutical Sciences, Utrecht, The Netherlands \\ 3 Netherlands Pharmacovigilance Foundation Lareb, 's-Hertogenbosch, The Netherlands \\ 4 Hospital Pharmacy Midden-Brabant, TweeSteden Hospital and St Elisabeth Hospital, \\ Tilburg, The Netherlands
}

\section{Abstract}

In sharp contrast with the pioneering days of pharmacovigilance, many countries around the world nowadays yearly collect case reports of suspected adverse reactions to medicines by the tens of thousands. The annual number of reports received by the WHO Uppsala Monitoring Centre (UMC) now exceeds $200000 .{ }^{[1]}$ Therefore, the traditional 'case by case' assessment of reports may at many centres no longer be feasible and there is a strong interest in automated screening programs for the identification of signals of possible importance in large electronic databases. In a nutshell, such programs quantitatively compare combina- tions of drugs and adverse events against the background of the database and use statistical parameters for a first signal selection. ${ }^{[2]}$ The advantages of automated quantitative signal generation are obvious. Unmanageably large amounts of data are transformed into portions of limited size, and perhaps particular focus, that can be overseen by the human mind. A concentration takes place of associations with increased probability of being signals. ${ }^{[2]}$ Powerful computers can do such calculations rapidly and minimal human time is needed. As an example, in the first quarter of 2001 the UMC has received case reports regarding alto- 
gether 55038 different drug-adverse event combinations. Of these, 19685 associations were more frequently reported than was expected from the background of the database and exceeded a pre-set quantitative threshold. Of these associations, 2109 had reached this threshold for the first time during that quarter. Of these 'new associations', 746 referred to WHO Adverse Reactions Terminology (WHOART) 'critical terms'. In this way, the original input of 55038 different reported drug-adverse event combinations was reduced to only 746 for further scrutiny.

In addition, data selection using statistical criteria is not influenced by prior knowledge and investigator's bias. Moreover it is objective, transparent and reproducible. Limitations are, on the other hand, that the selection mechanism is purely quantitative and does not take into account medical or pharmacological considerations, and that true connections that are not statistically prominent may be overlooked.

As defined previously, a signal in pharmacovigilance is more than just a statistical association. ${ }^{[3]}$ It consists of a hypothesis together with data and arguments; arguments in favour and against the hypothesis. These relate to numbers of cases, statistics, clinical medicine, pharmacology (kinetics, actions, previous knowledge) and epidemiology, and may also refer to findings with an experimental character. The discovery of a drug-induced disorder, from the earliest suspicion via a credible signal to a fully explained and understood phenomenon, is a lengthy process (figure 1). It may take years until the symptoms, frequency, mechanism and risk factors of an adverse reaction have been fully recognised and the causal connection has been definitely established. For many adverse reactions mentioned in the company's summary of product characteristics such questions have only partly been answered. They have been legally accepted but are scientifically still uncertain or incomplete. As a rule, a signal is a more or less strong suspicion. It is a 'snapshot', a picture of the situation on a given moment, somewhere in the course of discovery. Therefore, signals need follow-up with regard to both scientific credibility and clinical and regulatory relevance.

\section{Signal Detection}

Signal detection in pharmacovigilance comprises the processes of: selection of a drug-adverse event association of possible interest; the preliminary assessment of the available evidence; and a follow-up of how the signal develops. In the automated systems currently in use, the computer selects drug-adverse event pairs that stand out against the background of the database, according to prefixed statistical criteria, for example using the proportional reporting ratio (PRR) or, as is the case at the UMC, the information component (IC) calculated by a Bayesian confidence propagation neural network (BCPNN). ${ }^{[2]}$ Once the computer has at a given moment identified the associations that meet the quantitative criterion, individual assessors have to select those associations that deserve further preliminary attention. From now on the human mind has to decide whether the association is likely or not to represent a true signal and needs further examination. Criteria that often play a role in this selection process are listed in table I.

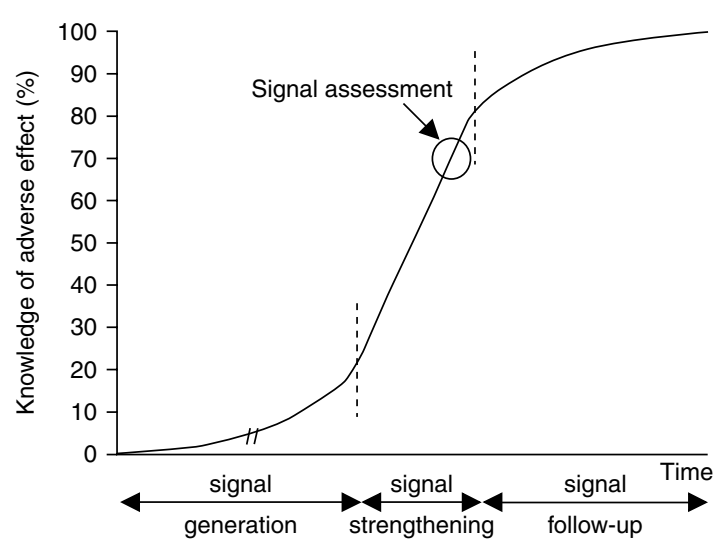

Fig. 1. The lengthy process of the discovery of a drug-induced disorder, from the earliest suspicion via a credible signal to a fully explained and understood phenomenon. 
Table I. Criteria for the selection of an association

\begin{tabular}{ll}
\hline Positive & Negative \\
\hline Unknown adverse reaction & Known (and labelled) \\
Strong statistical connection & Weak statistical connection \\
Unexpected & \\
$\begin{array}{l}\text { Expected but 'unlabelled' } \\
\text { Specific, characteristic }\end{array}$ & Unspecific, trivial event \\
Objective (definitive) event & Subjective event \\
$\begin{array}{l}\text { Typical drug-related event or } \\
\text { critical term }\end{array}$ & $\begin{array}{l}\text { Common disorder, e.g. } \\
\text { Low background frequency }\end{array}$ \\
Serious & High background frequency \\
High potential relevance & Not serious \\
\hline
\end{tabular}

Since a signal is by definition something new, first of all comes the question of whether or not the association has previously been recognised, i.e. labelled in the product information or described in the literature. In the future, when this assessment is done by the National Centres before the information is stored in the international database, this criterion will be automatically included in the routine output documents of the UMC (i.e. the Combination and Association Databases). In addition to the shift to statistical significance (a lower confidence interval value above zero in the UMC BCPNN system), the statistical position of the association in the database and its course over time provides valuable arguments for selecting or abandoning the association.

The next important consideration is how expected or unexpected the connection would be from the pharmacological point of view. Many new drugs are a close or distant relative of one or another - chemical, pharmacological or therapeutic - family (see table II). Such groups or subgroups often have a common profile as regards pharmacological actions (type A adverse effects), interactions, hypersensitivity reactions (type B adverse effects) or other problems (for example addiction). Understandably, but somewhat paradoxically, the situations that an adverse reaction is pharmacologically unexpected or expected (but 'unlabelled' in the Summary of Product Characteristics) can both be a reason for selecting the association.

A third major consideration concerns the clinical characteristics of the adverse event: is it something characteristic or non-specific, objective or subjective, rare or common (i.e. high background frequency), is it a typical drug reaction (for example agranulocytosis) or a recognised infectious or endogenous disorder?

Also the potential relevance of the association plays a role in the early decision to select an association for further attention. Reasons why a signal may be likely to be relevant are reviewed in table III. Pharmacovigilance has, first of all, an early warning function. New adverse drug reactions should be detected as soon as possible, in particular when they are a potential threat to public health or require for other reasons prompt regulatory action. Pharmaceutical companies in particular are concerned with unforeseen adverse reactions that

Table II. Examples of drug groups

\begin{tabular}{l}
\hline$\beta$-Lactam antibacterials \\
Amoxicillin \\
Cephalosporins \\
Carbapenems \\
Sulfonamides \\
Sulfonylureas \\
Thiazides \\
Quinine \\
(Hydroxyquinolines) \\
Aminoquinolines \\
Fluorquinolones \\
Nonsteroidal anti-inflammatory drugs \\
Opioids \\
Agonists \\
Antagonists \\
Benzodiazepines \\
Tricyclic compounds \\
Antipsychotics \\
Antihistamines \\
Antidepressants
\end{tabular}

Selective serotonin reuptake inhibitors

Anticonvulsants 
Table III. Reasons why a signal is likely to be relevant

Early warning
New adverse reaction; new drug
Public health issue
Important drug (serious indication; widely used)
Serious reaction
Large number of cases; rapid increase in reporting
Regulatory action; prevention
Change in merit/harm
Scientific or educational value
Strong evidence (credibility)

may change the effectiveness/risk balance of a drug, threatening its market position in comparison with competitive drugs. In addition, findings that are indicative of an unrecognised pharmacological action or other scientific finding, even though not a regulatory concern, may be pertinent to our knowledge of the drug and to the diagnosis and management of the patients. Also, since the case observations reported to a pharmacovigilance centre disclose the conditions under which adverse reactions occur in practice, they may have great educational value, even if the association may not be new or unknown. Obviously, also the credibility, i.e. the amount of evidence contained in the signal, contributes to the question whether a signal is likely to require - and to justify - rapid action.

\section{Signal Strengthening and Follow-Up}

As has already been said, a signal is a snapshot on a given moment in the lengthy process of the discovery of an adverse drug reaction (figure 1). Once a signal has been recognised and assessed, it needs to be followed how it evolves over time in the database, e.g. as regards absolute numbers of cases, the statistical parameters, exposure to the drug (utilisation) and the persistence of the characteristicity and consistency of the reporting pattern. Often further analysis of the database can provide a preliminary assessment of the strength of a signal (table IV). A comparison of the reporting patterns in different countries is a logic first step. A signal consisting of case reports from only one single country can be less convincing than one based on reports from for example 20 different countries. A 'best case-worst case' scenario can give a more balanced view of the strength of a signal. ${ }^{[4]} \mathrm{A}$ variety of comparisons, following from the hypothesis of the signal, using selections of the database (e.g. different drugs, drug groups or drug combinations) can produce a better picture of the statistical composition of the connection and of its consistency in different situations. A nested case control study can be an attractive way of doing an 'internal check' of a signal using the same database. ${ }^{[5]}$

A next step in signal follow-up is the use of data from other sources for a search for the presence of a similar connection (table V). Sometimes also experimental observations can be found that support the signal or provide a possible explanation, e.g. in individual case reports (see section 4) or in the registration file of the company.

\section{Signals not Found by Disproportionality}

A signal may consist of only a few case reports and not be statistically prominent and nevertheless herald a true adverse reaction. This may in particular occur when the event is a typically druginduced disorder, for instance agranulocytosis or erythema multiforme, and is relatively frequently reported and 'over-represented' in the database. A recent example are anaphylactic reactions to omeprazole and other proton pump inhibitors. ${ }^{[6]}$ In the current system of the UMC such signals will not be

Table IV. Signal follow-up (same database)

Development over time of the quantitative data and of the
consistency of the pattern
Signal strengthening
Individual case assessment
Country (source) comparisons
'Best case-worst case' scenario
Targeted comparisons
Nested case control studies


Table V. Signal follow-up (other databases and sources)

Presence of a similar connection

Additional clinical observations (e.g. literature, spontaneous reporting, registration file)

Experimental data (e.g. pharmacological, immunological)

detected through the so-called Associations Database. However, they can be found in the Combinations Database, which includes all new combinations, statistically prominent or not. Although the results of the BCPNN signalling system of the $\mathrm{UMC}^{[7]}$ and of systems in place at other centres show reasonable sensitivity and specificity, they should not be regarded as a panacea.

\section{The Balance of Evidence in a Signal (Credibility)}

It is a highly professional and characteristic feature of pharmacovigilance that during signal follow-up on the right moment the balance must be made of the - usually incomplete - evidence, in favour and against the connection, to decide the strength of the suspicion. This evaluation is a combined assessment of the individual reports 'case by case', of the aggregated data (clinically and statistically), and of information from other sources. Building on the original thoughts of Bradford-Hill, a number of quantitative and qualitative criteria have been identified that can give structure to the process (table VI). ${ }^{[3]}$ For obvious reasons there is some repetition of the criteria in table VI and those already listed in table I and table III. 'At the right moment' refers to a delicate weighing of the credibility of the signal, its importance for individual users and in the public health perspective, of the measures needed, and of the consequences if the signal proves false.

In addition to the quantitative strength of the association, the existence of a characteristic and consistent pattern in the reported events can be a strong argument in favour of a connection.
As always in pharmacology, the presence of a dose-response relationship - i.e. a more frequent or more severe effect when high doses are used can represent substantial evidence. Often the availability of a plausible hypothesis as regard the possible pharmacological or pathological mechanism can add to the credibility, although with adverse reactions, where often unidentified factors play a role, this argument can be a poor guide. Deliberate experiments in humans are rarely justified. Nevertheless, in case reports often data elements can be found that have an experimental aspect; for example the course of events after stopping the drug ('dechallenge') or after an (often inadvertent) reexposure, or information regarding blood levels, metabolites, or perhaps a drug-dependent immunological phenomenon. Pharmacokinetic or laboratory findings can be suggestive of a possible mechanism (e.g. a drug interaction). An again different but often helpful piece of information can be if there is a previous experience with a drug that may serve as an analogy. Finally there is the often very influential issue of the nature of the event (objectivity, specificity), the quality of the documentation of the observation, and the results of standardised causality assessment of individual case reports. ${ }^{[8]}$

Table VI. The balance of evidence in a signal

Quantitative strength of the association

Number of case reports

Statistical disproportionality

Consistency of the data (pattern)

Exposure-response relationship

Site, timing, dose, reversibility

Biological plausibility of hypothesis

Pharmacological, pathological

Experimental findings

e.g. dechallenge, rechallange, blood levels, metabolites, drug-dependent antibodies

\section{Analogies}

Nature and quality of the data

Objectivity, documentation, causality assessment 


\section{Signal Testing}

Time and again there has been confusion, for regulators, companies or the media, following from the uncertain nature of signals generated by spontaneous reporting and the inherent limitations of the system to produce secure and quantitative information. Spontaneous reporting has been designed as a system for hypothesis generation in the first place. As a rule, further study, using the most appropriate (and usually different) method, is needed to put the hypothesis to the test. ${ }^{[7]}$ Often this is a formal pharmacoepidemiological study, but also pharmacological or pathological studies may give support. Nowadays, on a worldwide basis there are far more signals found than can reasonably be tested. Further pharmacoepidemiological studies take time and money, and in the case of serious problems measures need to be taken without delay. ${ }^{[9]}$ More comprehensive pharmacoepidmiological study programs may improve signal testing and add to the scientific basis of post-approval regulatory decision making.

\section{From Signal to Action}

As is reviewed in table VII, signals in pharmacovigilance can play a role in a variety of activities, ranging from simple and preliminary communications to regulatory actions and scientific publications. The WHO-UMC international pharmacovigilance system is first of all a source of signals to be tested and evaluated in further studies by national agencies and pharmaceutical companies. In the years to come a wider use is envisaged of the WHO-UMC database, with a more intensive col-

Table VII. From signal to action

Communication of information (National Centres, company, other institutes)

Initiation of further study (hypothesis testing), e.g. follow-up study, cross sectional study

Regulatory action (e.g. data sheet change)

Publication (newsletter, article)
Table VIII. A future combined quantitative and qualitative automated signal detection system?

Quantitative data, e.g. absolute number of cases; level or disproportionality; increase in disproportionality (simple combinations; interactions; complex patterns)

Source distribution, e.g. number of reporters or countries Novelty, e.g. first report, statistical strength, 'unlabelled' Importance/regulatory, e.g. serious, critical term, malformation, fatal

Clinical, e.g. typical adverse drug reaction, objective event, low spontaneous frequency

Course, e.g. outcome plausible, dechallenge, rechallenge

Number of suspected drugs per case

Documentation quality grading

Standardised case causality assessment

laboration with academic pharmacoepidemiology around the world.

\section{Future Developments in Automated Signal Generation}

Besides the statistical data, many of the other criteria and considerations reviewed can be categorised, either as different qualities (e.g. serious versus non-serious) or in a semiquantitative way, and be stored as such in a database. Likewise they could be incorporated in a future automated signal generation system above (see table VIII). Although their distribution is restricted, the comprehensive Combinations and Association Databases regularly produced by the UMC are already a step towards such a system. More complex future systems, integrating several criteria of different nature, should be capable of selecting signals with a considerably higher likelihood of importance and credibility and enabling easy and flexible early signal strengthening.

\section{Acknowledgements}

We gratefully acknowledge the valuable discussions regarding signal detection during recent meetings of the Uppsala Monitoring Centre Signal Review Panel. 


\section{References}

1. Olsson S. The role of the WHO programme on international drug monitoring in coordinating worldwide drug safety efforts. Drug Saf 1998; 19: 1-10

2. Bate A, Lindquist M, Edwards IR, et al. A Bayesian neural network method for adverse drug reaction signal generation. Eur J Clin Pharmacol 1998; 54: 315-21

3. Meyboom RHB, Egberts ACG, Edwards IR, et al. Principles of signal detection in pharmacovigilance. Drug Saf 1997; 16: 355-65

4. Bate A, Lindquist M, Orre R, et al. Data mining analyses of pharmacovigilance signals in relation to relevant comparison drugs. Eur J Clin Pharmacol. In press

5. Egberts ACG, Meyboom RHB, De Koning FHP, et al. Nonpuerperal lactation associated with antidepressant drug use. Br J Clin Pharmacol 1997; 44: 277-81

6. Natsch S, Vinks MHAM, Voogt AK, et al. Anaphylactic reactions to proton-pump inhibitors. Ann Pharmacother 2000; 34 : 474-6
7. Lindquist M, Ståhl M, Bate A, et al. A retrospective evaluation of a data mining approach to aid finding new adverse drug reaction signals in the WHO international database. Drug Saf 2000; $23: 533-42$

8. Meyboom RHB, Egberts ACG, Gribnau FWJ, et al. Causal or casual?. The role of causality assessment in pharmacovigilance. Drug Saf 1997; 16: 374-89

9. Meyboom RHB, Hekster YA, Egberts ACG, et al. Pharmacovigilance in perspective. Drug Saf 1997; 21: 429-47

Correspondence and offprints: Dr Ronald H.B. Meyboom, The Uppsala Monitoring Centre, Stora Torget 3, Uppsala, S-753 20, Sweden.

E-mail: r.meyboom@who-umc.org 